\title{
The Laser calibration system for LHAASO-WFCTA
}

\author{
Y. Wang, ${ }^{a, *}$ L. Chen, ${ }^{a}$ H. Liu, ${ }^{a}{ }^{\text {Q.N. Sun, }}{ }^{a}$ X. Li, ${ }^{a}$ J.J. Xia, ${ }^{a}$ F.R. Zhu, ${ }^{a}$ L.S. Geng, ${ }^{b}$ S.S. \\ Zhang $^{b}$ and $Y$. Zhang ${ }^{b}$ on behalf of the LHAASO Collaboration \\ (a complete list of authors can be found at the end of the proceedings) \\ ${ }^{a}$ School of Physical Science and Technology, \\ Southwest Jiaotong University, Chengdu 611756, China \\ ${ }^{b}$ Key Laboratory of Particle Astrophysics, \\ Institute of High Energy Physics of the Chinese Academy of Sciences, Beijing 100049, China \\ E-mail: yangwang@swjtu.edu.cn
}

The Wide Field-of-view Cherenkov Telescope Array (WFCTA) of Large High Altitude Air Shower Observatory (LHAASO) is designed to measure the Cherenkov and fluorescent light created in the extensive air showers by cosmic rays (CRs) from $\sim 30 \mathrm{TeV}$ to several EeV. In order to achieve an end-to-end calibration method for WFCTA, three Laser lidar facilities have been installed at LHAASO. They feature two $\mathrm{N}_{2}$ and one YAG Lasers which direct calibrated and pulsed beams into sky. Light scattered from Laser beams produces tracks recorded by the telescope detectors. The Laser calibration systems are used to investigate properties of the atmosphere and air fluorescence. In this paper, we introduce the design and performance of the Laser facilities at an altitude of 4410 $\mathrm{m}$. Great cares were taken to select the data collected by telescopes at clear nights for our analysis. After excluding the inconsistency of all SiPMs of the telescope, we find that the experimental data of the images collected by telescopes agree noticeably well with Monte Carlo's simulation.

$37^{\text {th }}$ International Cosmic Ray Conference (ICRC 2021)

July 12th-23rd, 2021

Online - Berlin, Germany

\footnotetext{
*Presenter
} 


\section{Introduction}

Large High Altitude Air Shower Observatory (LHAASO) is a dual-task facility designed for cosmic-ray (CR) and $\gamma$-ray studies with an energy up to petaelectronvolt ( $\mathrm{PeV} ; 10^{15}$ electronvolts)[1]. It consists of three interconnected arrays of - the Water Cherenkov Detector Array (WCDA), Kilometer Square Array (KM2A) and Wide Field-of-view Cherenkov Telescope Array (WFCTA). LHAASO is located at 4,410 m above sea level in Haizi Mountain, Sichuan Province, China [2]. The WFCTA is designed to measure the Cherenkov and fluorescent light created in the extensive air showers by $\mathrm{CRs}$ from $\sim 30 \mathrm{TeV}$ to several EeV. It is essential to find a complete and self-consistent observation over this whole energy range by a precise measurement of the spectrum at LHAASO with sufficient overlap between different energy regions, which is the scientific goal of the WFCTA. The energy spectra of cosmic rays measured by WFCTA are mainly obtained by calculating the number of photons detected by the telescope. As a result, it is necessary to apply an end-to-end calibration method to the number of photons, considering the overall conversion factor from analog-to-digital converter (ADC) counts to the light flux at the telescope entrance. The ambiguity of the end-to-end calibration originates from the light energy probing, the light calculation concerning Rayleigh and aerosol scattering models, as well as ADC counts. Cherenkov light is partially lost due to the scattering by air molecules (Rayleigh scattering) and aerosols (Mie scattering) when propagating in air[3, 4]. The lost caused by Rayleigh scattering can be retrieved by a theoretic calculation. In contrast, the lost by aerosol scattering can only be estimated by experimental measurement. The scattered light attributable to molecular and aerosol components produces tracks which have several features in common with the ones that originate from extensive air showers[5]. The key point in CR energy spectra measurement is the determination of the total energy of reconstructed air showers as it reqiures an absolute calibration for the detector. The using of fast Laser pulses would make the absolute calibration of the telescopes more forthright and reliable[6]. The Laser calibration system (LCS) is subsequently adopted to detect the atmospheric fluorescence (Cherenkov light). After propagating in air, photons from the Laser pulses were detected by telescopes along the track. Based upon the light flux calculation at the front of the detector, one can straightly calibrate the detector by analyzing the measured ADC counts[7]. The LCS was designed to achieve two goals, including monitoring atmospheric conditions, and improving the geometric reconstruction of air shower events. We will discuss each part of the LCS in detail in this paper.

\section{System Overview}

The LCS of WFCTA is generally composed of two parts: hardware and software for controlling, including a Laser head and its controller, a calibration room, the cruise control and imaging system. For the WFCTA project, in response to various needs, 3 sets of Laser calibration and atmospheric monitoring systems have been built at LHAASO, as shown in Figure 1. The L2 is $465 \mathrm{~m}$, L3 is $1075 \mathrm{~m}$ and L4 is $650 \mathrm{~m}$ from the telescope array, respectively. L1, which is not shown in Figure 1 , is principally used to measure the attenuation due to both absorptive and scattering processes on their way to the detector, and is under construction currently.

The principle of the absolute calibration of photons for a telescope is as follows: a Laser pulse with a certain energy and polarization is emitted from the calibration room to the field of view 


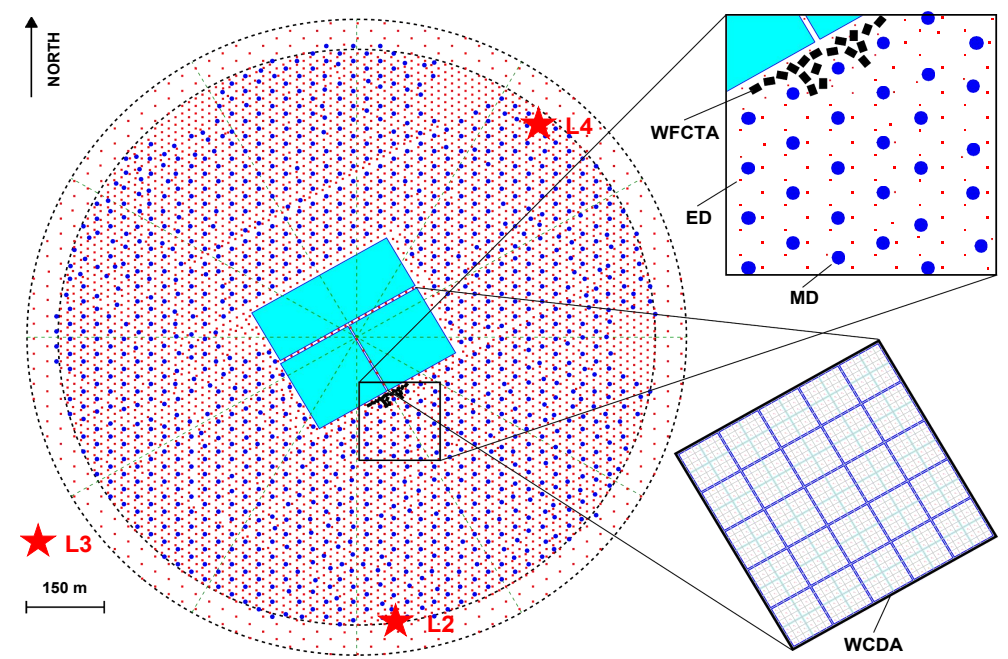

Figure 1: The schematic layout of LHAASO. The location of each LCS is indicated by a red star (L2, L3 and L4).

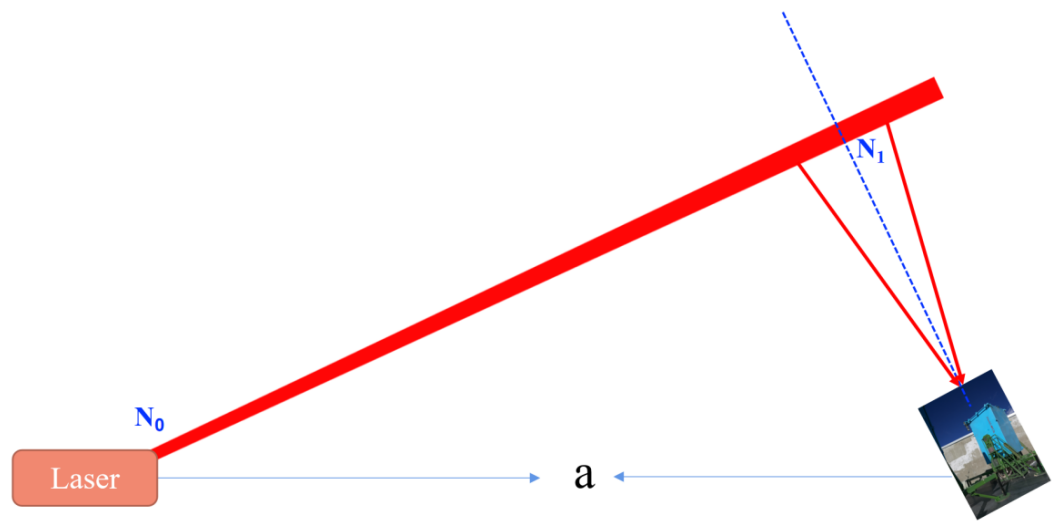

Figure 2: The schematic diagram of Laser calibration: $\mathrm{N}_{0}$ and $\mathrm{N}_{1}$ are the number of photons emitted into atmosphere and scattered into the telescope, respectively, and a is the distance between a Laser and the telescope.

(FoV) of the telescope at a specific angle with a time delay during a period of time (see Fig. 2). The scattering lights will trigger tubes and the direction of the Laser track will be recorded by the electronics system when a Laser pulse passes the FoV of the telescope [8]. By the calculation of the expected and the acquired light fluxes at the front of the aperture of the telescope, it is attainable to calibrate the number of photons from a Cherenkov light induced in air showers by CRs as shown in Figure 2. To minimize the scattering by the aerosol, the calibration is always carrying out at very clear nights. The LCSs are an unstaffed facilities and they are operated remotely via the internet by computers in office. The layout of L2 is depicted in Figure 3. It mainly consists of six parts, including a computer controlling system, a mechanical system, a temperature controlling system, a timing system, a monitoring system and a nitrogen Laser. A programmable logic controller (PLC) is used to precisely and remotely control the accuracy of the Laser beam pointing through a high-precision rotating and lifting platform. Besides, a home-made thermotank was designed to 
control the temperature and humidity to improve the performance and stability of the Laser system, which results in a thermal fluctuation of less than $1{ }^{\circ} \mathrm{C}$ inside the container even in the winter at an altitude of $4410 \mathrm{~m}$ at LHAASO. The timing system, the Global Position System (GPS), provides a daily time calibration for the computer and a signal to trigger the Laser and control the time delay between them. The monitoring system is continuously recording the temperature and humidity in the room. The measurement shows the angular and lifting accuracy of the mechanical platform is better than $0.004^{\circ}$ and $0.075 \mathrm{~mm}$, respectively. In addition, the corresponding repeatability of this platform is better than $0.003^{\circ}$ and $0.025 \mathrm{~mm}$, respectively. All of these parameters permits a stable and accurate pointing of the Laser beam into sky. The heart of the system is a nitrogen Laser (NL100), manufactured by Stanford Research Systems Inc., which produces a circularly polarized beam. It is triggered by the GPS signal and provides $3.5 \mathrm{~ns}$ pulses at $337 \mathrm{~nm}$, with a repetition rates of $1 \mathrm{~Hz}$. The pulse energy is up to $170 \mu \mathrm{J}$. An energy detector (PE50-DIF-C, StarLab Inc.) with a damaging threshold of $1 \mathrm{~J} / \mathrm{cm}^{2}$ measures the pulse energy.

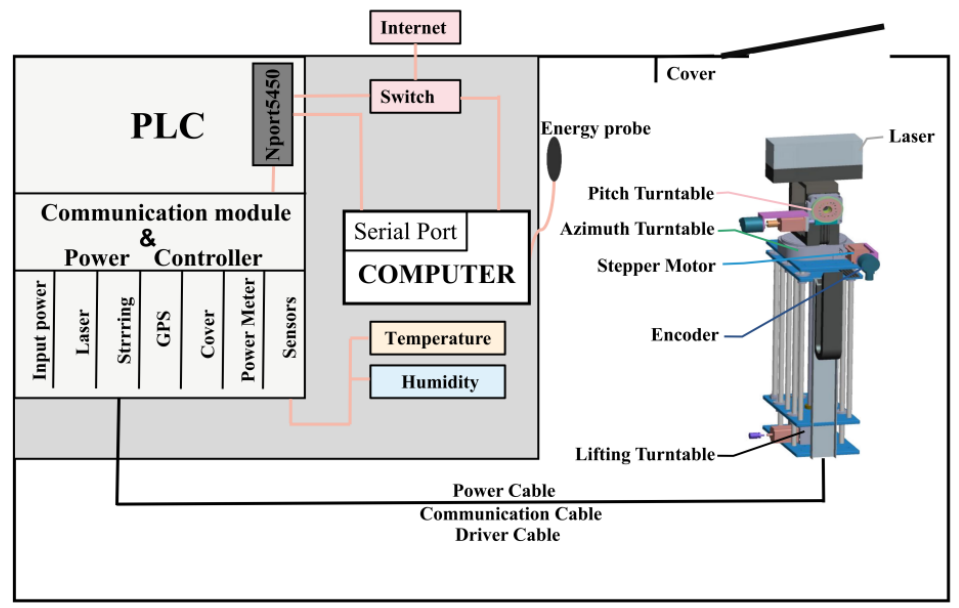

Figure 3: Schematic diagram of the $\mathrm{L} 2 \mathrm{~N}_{2}$ Laser facility.

In order to increase the number of photons with different wavelength, a frequency tripled $\mathrm{Nd}$ : YAG (Ultra 20) Laser was set up at the site of L3, manufactured by Quantel Inc., which produces a vertically and linearly polarized beam of $355 \mathrm{~nm}$. The beam is pulsed, with a width of $7 \mathrm{~ns}$, and a maximum energy per pulse of $2 \mathrm{~mJ}$. In addition to the $355 \mathrm{~nm}$ light, the Laser head produces a second harmonic of $1064 \mathrm{~nm}$. To remove this unwanted component and the residual light of 1064 $\mathrm{nm}$, a separator was used to reflect only the desired $355 \mathrm{~nm}$ component. The spectral purity of the beam is thus better than $99.9 \%$. Despite that the YAG Laser generates linearly polarized beam, it is favorable to depolarize the beam leaving the LCS as such a beam will, when emitted vertically, scatter equal amounts of light in the field-of-view direction[9]. Therefore, a quarter-wave plate was brought in to the beam path and a phaseshift was added to convert the linear polarization into a circular one. The net polarization of the laser beam is then within $8 \%$ of random. If the measured error (RMS) of the pulse energy of a YAG Laser is less than $2 \%$, then the calculated error of a Rayligh's scattered intensity will be less than $3 \%$. As a result, an error less that $5 \%$ is feasible for 
the calibration of the photon number. Therefore, it is of great importance to accurately monitor and measure each laser pulse to minimize the error attributed to the energy fluctuation. A beam splitter was added in the beam path and reflected $\sim 40 \%$ of the beam (reference beam) into an energy detector. The transmitted beam (calibration beam) was directed by another seven mirrors into sky and the orientation was regulated by a rotational stage. The ratio of intensity between the calibration and reference beam is given by:

$$
\frac{I_{\mathrm{cal}}}{I_{\text {ref }}}=\frac{1-R_{0}}{R_{0}} R_{1} R_{2} R_{3} R_{4} R_{5} R_{6} R_{7}
$$

where $R_{0}$ and $R_{i}(i=1,2, \ldots 7)$ are the reflectivity of the beamsplitter and the mirror, respectively. And $I_{\text {ref }}$ and $I_{\text {cal }}$ are the intensity of reference and calibration beam, respectively. The ratio is independent of the emitted Laser pulse energy according to formula (1) and only committed to the angle of incidence and the polarization of the beam. The fluctuation of the emitted light cancels out and it paves the way to a pulse-by-pulse energy monitoring by recording the pulse energy of the reference beam.

During the calibration procedure, the Laser beam emits into sky and is aimed at the FoV of the telescope. The WFCTA of LHAASO measures shower characteristics using the Cherenkov light produced by secondary particles generated in the air. Eighteen wide field of view Cherenkov telescopes were installed surveying the sky above the whole array with a coverage of 4608 square degrees. The telescopes have aluminized spherical mirrors with an area of $5 \mathrm{~m}^{2}$, protected by a layer of $\mathrm{MgF}_{2}$ coating. It can be tilted up and down in elevation from $0^{\circ}$ to $90^{\circ}$. A camera with 32 $\times 32$ square pixels was installed in the focal plane of the telescope, $2870 \mathrm{~mm}$ away from the mirror center, and it achieves a $16^{\circ} \times 16^{\circ} \mathrm{FoV}$. The Cherenkov light is collected by the spherical mirror and then reflected to a silicon photomultiplier (SiPM) imaging detector, where it becomes a fluorescence or Cherenkov image. The SiPM is an array of 0.36 million avalanche photo diodes (APD) with a micro-cell size of $25 \mu \mathrm{m}$, providing a dynamic range from 10 to 40,000 photo-electrons with a measured non-linearity less than $5 \%[5,10,11]$.
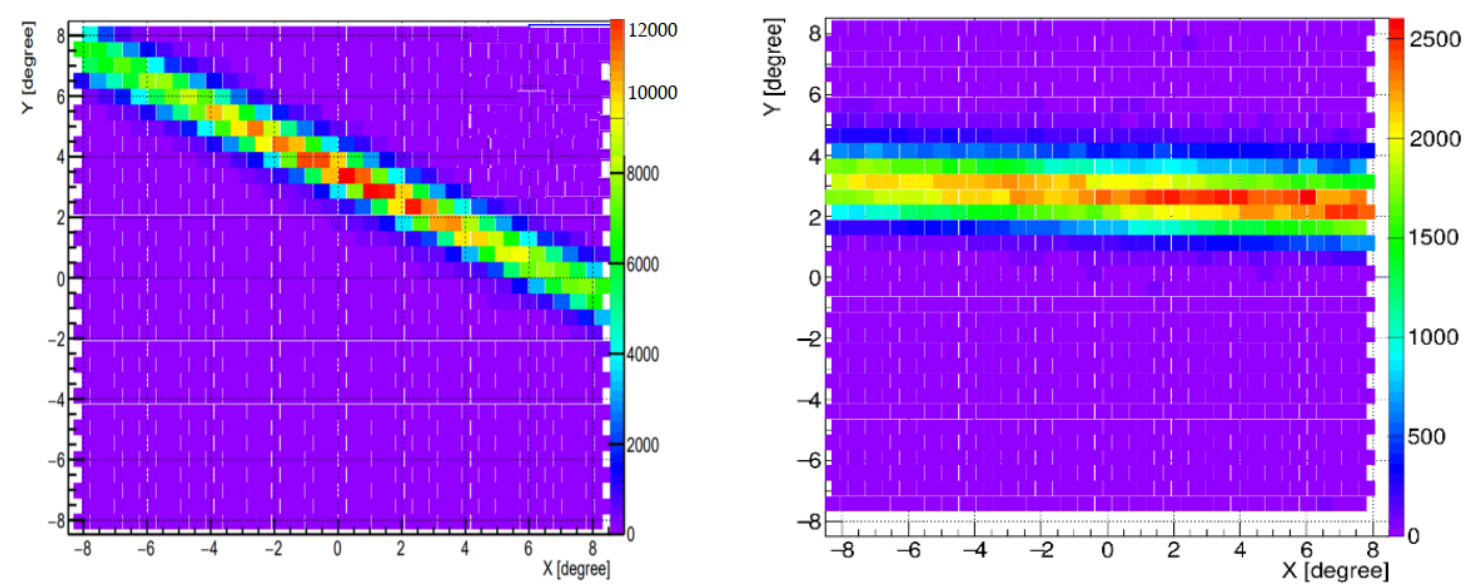

Figure 4: The images detected by a telescope of WFCTA for a nitrogen Laser event (left) and a YAG Laser event (right).

Most of the tracks recorded by the telescope and it is of great consequence to separate these tracks from those produced by cosmic ray air showers. The Laser is fired at specific times that have 

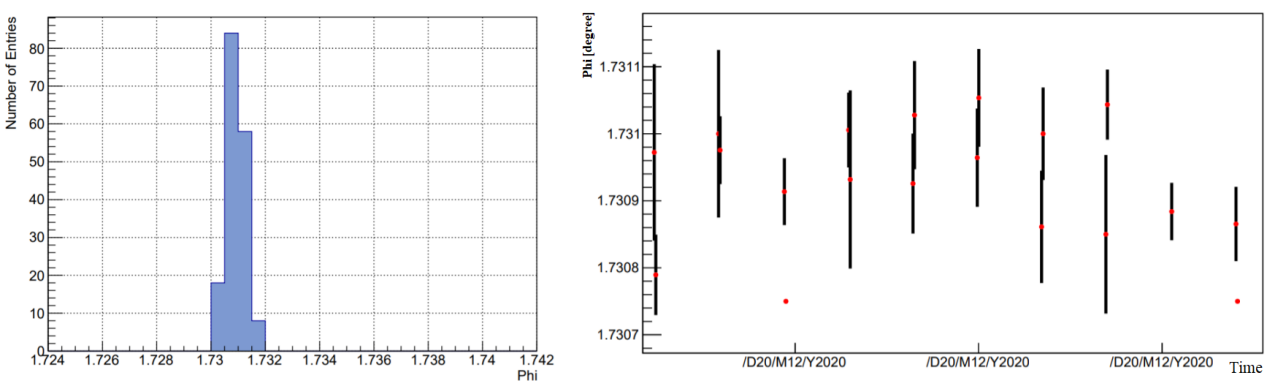

Figure 5: Left: the mean of phi in one minute vs Time; Right: the distribution of phi over one night.

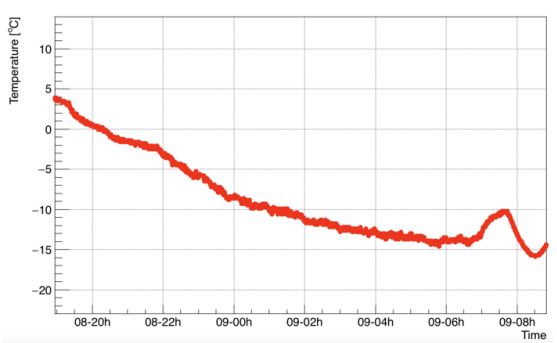

(a)

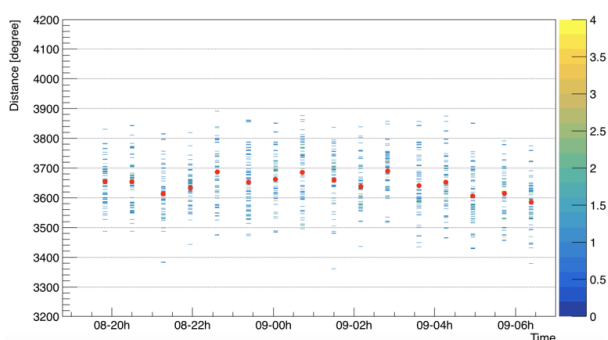

(b)

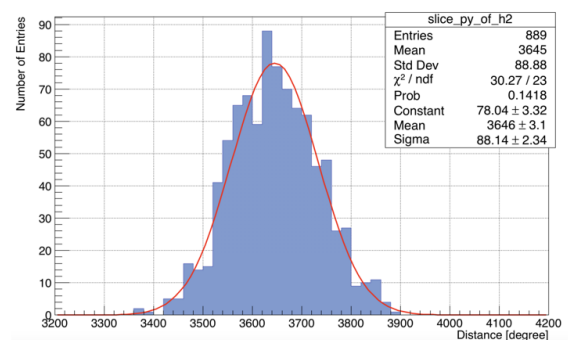

(c)

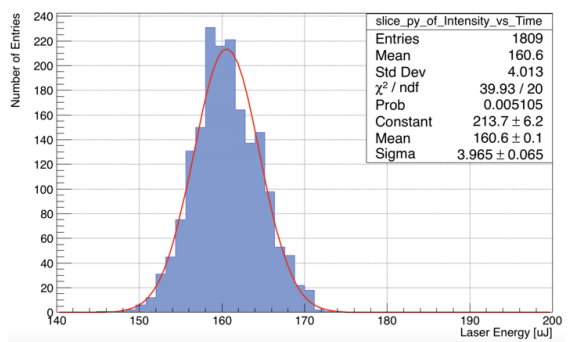

(d)

Figure 6: Left: Temperature vs Time (a) and Npe vs Time (b); Right: distribution of the Npe (c) and distribution of laser energy (d).

a fixed offset from the start of the second as defined by the GPS. In the experiment, a Laser pulse with a repetition rate of $1 \mathrm{~Hz}$ is triggered externally by a calibrated GPS signal and the same GPS signal is also provided to the WFCTA system so as to find the Laser tracks from the telescope. Each Laser acquires external trigger signals with different delays, intending to distinguish tracks from different Laser systems. Two images detected by a telescope of WFCTA for a nitrogen Laser event and a YAG Laser event, respectively, are shown in Figure 4. To study the geometrical stability of the Laser system, the image detected by the telescope is parameterized with formula (2), where CC and phi are two parameters to describe the slope and intercept of the image. The distribution of phi at one night and the mean value in one minute versus time is shown in Figure 5.

$$
X * \sin (p h i)-Y * \cos (p h i)+C C=0
$$

The rapid change of temperature at LHAASO over one night is relatively large (it covers from $5{ }^{\circ} \mathrm{C}$ to $-30^{\circ} \mathrm{C}$ ), and the gain of SiPMs is changing with the temperature. The number of photon-electron (Npe) of nitrogen Laser event detected by one telescope is shown in Figure 6 to study the effect of 

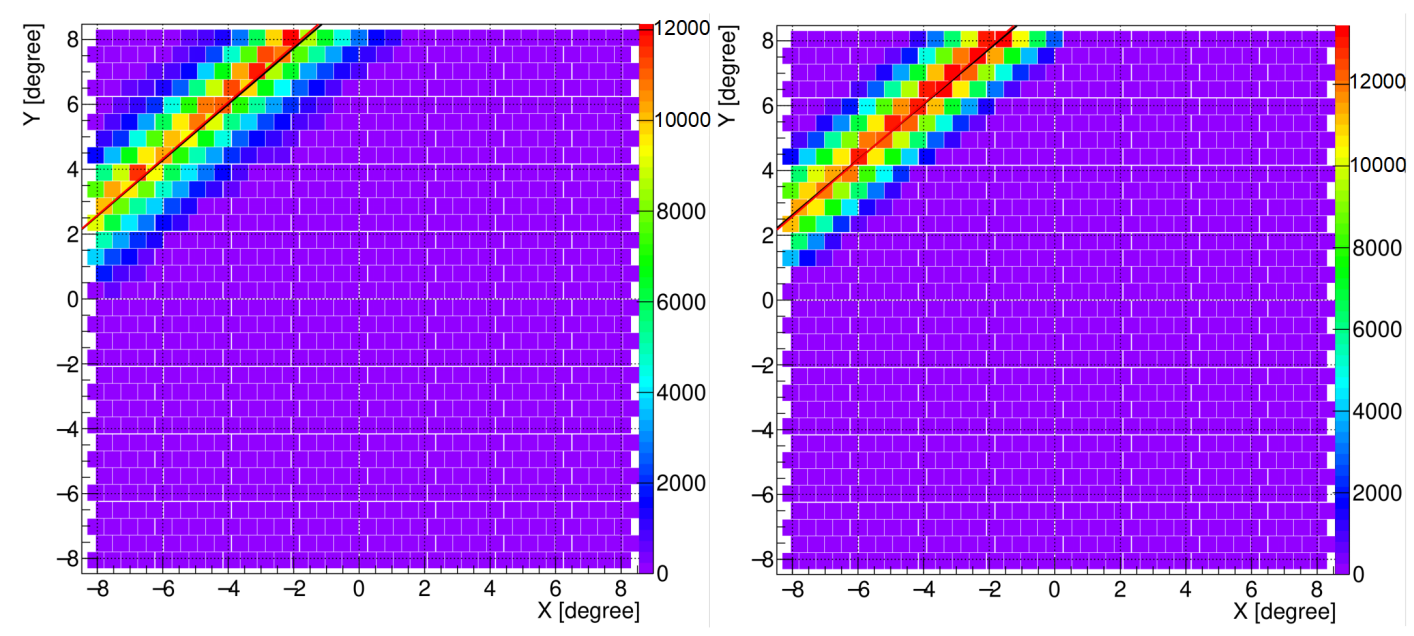

Figure 7: The image of one Laser event detected by one telescope of WFCTA. Left: experimental data; Right: Monte Carlo simulated event. The black line is a linear fit for the image, and the red line is the predicted position of the image based on the calculation with the astronomical package slalib, which is the same for the plots both on the left and right.

the temperature on LCS. After the correction of temperature on Npe (based on a carefully designed LED system), the mean Npe is a constant as temperature varies. Moreover, the fluctuations of Npe are very similar to that of the pulse energy, which means that the fluctuation introduced by the Laser energy instability is considerably small. A dedicated program based on Monte Carlo method was developed to simulate the LCS, including the properties of the following parameters: the Laser ( the energy, polarization, beam size and divergence, etc.) and the rotating and lifting platform (the absolute zero direction and the elevation angle of the rotating axis, etc). The Mie scattering in the atmosphere and the simulation inside the telescope (the same one for the CR simulation) are also involved. Currently the US standard model is applied to the simulation, and other atmosphere models are also optional. Figure 7 illustrates the comparison of the experimental data of the images collected by telescopes with the Monte Carlo simulated events, which agrees well. Detailed studies on the gain of the telescope and aerosol measurement are ongoing.

\section{Summary}

Three Laser systems have been disployed at LHAASO : two $\mathrm{N}_{2}$ Lasers have been operating continuously since October 2019, as well as a YAG Laser since December 2020. The precise control of the temperature and rotational stage allowed us to minimize the fluctuation and pointing instability of the Laser pulses, which is critical for the WFCTA. The tracks from both the $\mathrm{N}_{2}$ and YAG Lasers were recorded by the telescopes. The analysis exhibits a good agreement between the experimental and simulated data. Further studies are ongoing.

Acknowledgements We are grateful to all members who work for LHAASO project. This work is supported in China by the Fundamental Research Funds for the Central Universities (grant numbers 2682020CX77, 2682020CX73, 2682020CX74). It is also supported by the Science and Technology Department of Sichuan Province (grant numbers 2021YFSY0031, 2020YFSY0016), 
and by NSFC (grant number 11947404), and by National Key R\&D program of China (grant number 2018YFA0404201).

\section{References}

[1] Cao, Z., Aharonian, F.A., An, Q. et al., Ultrahigh-energy photons up to 1.4 petaelectronvolts from $12 \gamma$-ray Galactic sources, Nature 594, 33-36 (2021).

[2] Cao, Z, A future project at Tibet: the large high altitude air shower observatory (LHAASO), Chin. Phys C 34, 249-252 (2010).

[3] G. Placzek, The Rayleigh and Raman Scattering, Lawrence Radiation Laboratory (1959).

[4] G. Mie, Contributions to the optics of turbid media, particularly of colloidal metal solutions, Ann. Phys 25 (3), 377-445 (1908).

[5] F. Aharonian, Q. An, Axikegu, L. X. Bai, Y. X. Bai, Y. W. Bao, D. Bastieri, X. J. Bi, Y. J. Bi, H. Cai et al., Calibration of the Air Shower Energy Scale of the Water and Air Cherenkov Techniques in the LHAASO experiment, [arXiv:2104.04965 [astro-ph.IM]] (2020).

[6] J.L.Liu, F.R. Zhu, H.Y. Jia, Y.X. Bai, Z. Cao, M.J. Chen, S.Z. Chen, S.H. Feng, B. Gao, M.H. Gu, H.H. He, C. Hou, J. Liu, L.L. Ma, G. Xiao, M. Zha, B.K. Zhang, S.S. Zhang, Y. Zhang, J. Zhao, B. Zhou, X. Zuo, A calibration of WFCTA prototype telescopes using $\mathrm{N}_{2}$, Nuclear Inst. and Methods in Physics Research, A 877, 278-287 (2018).

[7] A. Konopelkoa et al., Properties and performance of two wide field of view Cherenkov/fluorescence telescope array prototypes, Nuclear Inst. and Methods in Physics Research, A 629, 57-65 (2011).

[8] Cao, Z, The HEGRA Collaboration, Detection of gamma rays above $1 \mathrm{TeV}$ from the Crab Nebula by the second HEGRA imaging atmospheric Cherenkov telescope at La Palma, Astropart. Phys. 4 (3), 199-215 (1996).

[9] B. Fick, M. Malek, J. A. J. Matthews, The Central Laser Facility at the Pierre Auger Observatory, JINST 1, P11003 (2006).

[10] B. Bi, S.S. Zhang et al., Performance of SiPMs and pre-amplifier for the wide field of view Cherenkov telescope array of LHAASO, Nucl. Instrum. Methods Phys. Res. A899, 94-100 (2018).

[11] Cao, Z. et al., Introduction to Large High Altitude Air Shower Observatory (LHAASO), Chin.Astron.Astrophys. 43, 457-478 (2019). 


\section{Full Authors List: LHAASO Collaboration}

Zhen $\mathrm{Cao}^{1,2,3}$, F. Aharonian ${ }^{4,5}$, Q. An ${ }^{6,7}$, Axikegu ${ }^{8}$, L.X. Bai ${ }^{9}$, Y.X. Bai ${ }^{1,3}$, L.X. Bai ${ }^{9}$, Y.X. Bai ${ }^{1,3}$, Y.W. Bao ${ }^{10}$, D. Bastieri ${ }^{11}$, X.J. Bi ${ }^{1,2,3}$, Y.J. Bi ${ }^{1,3}$, H. Cai ${ }^{12}$, J.T. Cai ${ }^{11}$, Zhe Cao ${ }^{6,7}$, J. Chang ${ }^{13}$, J.F. Chang ${ }^{1,3,6}$, B.M. Chen ${ }^{14}$, E.S. Chen ${ }^{1,2,3}$, J. Chen ${ }^{9}$, Liang Chen $^{1,2,3}$, Liang Chen ${ }^{15}$, Long Chen ${ }^{8}$, M.J. Chen ${ }^{1,3}$, M.L. Chen ${ }^{1,3,6}$, Q.H. Chen ${ }^{8}$, S.H. Chen ${ }^{1,2,3}$, S.Z. Chen ${ }^{1,3}$, T.L. Chen ${ }^{16}$,X.L. $\mathrm{Chen}^{1,2,3}$, Y. Chen $^{10}$, N. Cheng ${ }^{1,3}$, Y.D. Cheng ${ }^{1,3}$, S.W. Cui ${ }^{14}$, X.H. Cuii ${ }^{17}$, Y.D. Cui ${ }^{18}$, B. D'Ettorre Piazzoli ${ }^{19}$, B.Z. Dai ${ }^{20}$, H.L. Dai $^{1,3,6}$, Z.G. Dai ${ }^{7}$, Danzengluobu ${ }^{16}$, D. della Volpe ${ }^{21}$, X.J. Dong ${ }^{1,3}$, K.K. Duan ${ }^{13}$, J.H. Fan ${ }^{11}$, Y.Z. Fan ${ }^{13}$, Z.X. Fan ${ }^{1,3}$, J. Fang ${ }^{20}$, K. Fang $^{1,3}$, C.F. Feng ${ }^{22}$, L. Feng ${ }^{13}$, S.H. Feng ${ }^{1,3}$, Y.L. Feng ${ }^{13}$, B. Gao ${ }^{1,3}$, C.D. Gao ${ }^{22}$, L.Q. Gao ${ }^{1,2,3}$, Q. Gao ${ }^{16}$, W. Gao ${ }^{22}$, M.M. Ge $^{20}$, L.S. Geng ${ }^{1,3}$, G.H. Gong ${ }^{23}$, Q.B. Gou ${ }^{1,3}$, M.H. Gu${ }^{1,3,6}$, F.L. Guo ${ }^{15}$, J.G. Guo ${ }^{1,2,3}$, X.L. Guo ${ }^{8}$, Y.Q. Guo ${ }^{1,3}$, Y.Y. Guo ${ }^{1,2,3,13}$, Y.A. $\mathrm{Han}^{24}$, H.H. He $\mathrm{H}^{1,2,3}$, H.N. He ${ }^{13}$, J.C. He ${ }^{1,2,3}$, S.L. He ${ }^{11}$, X.B. He ${ }^{18}$, Y. He ${ }^{8}$, M. Heller ${ }^{21}$, Y.K. Hor ${ }^{18}$, C. Hou ${ }^{1,3}$, H.B. Hu ${ }^{1,2,3}$, S.

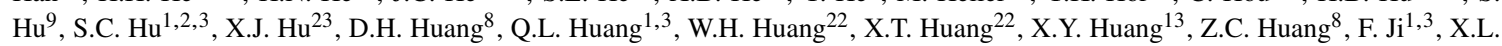
$\mathrm{Ji}^{1,3,6}$, H.Y. Jia ${ }^{8}$, K. Jiang ${ }^{6,7}$, Z.J. Jiang ${ }^{20}$, C. Jin ${ }^{1,2,3}$, T. Ke ${ }^{1,3}$, D. Kuleshov ${ }^{25}$, K. Levochkin ${ }^{25}$, B.B. Li ${ }^{14}$, Cheng Li ${ }^{6,7}$, Cong Li ${ }^{1,3}$,

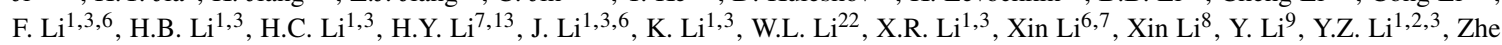

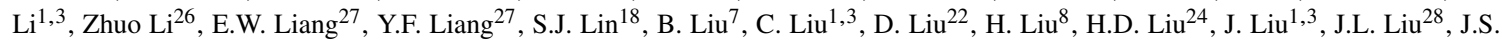

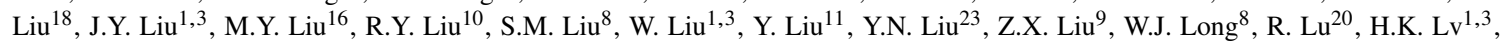

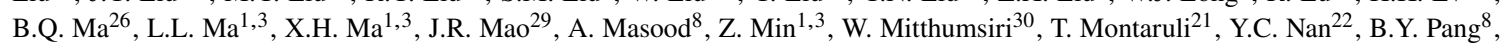
P. Pattarakijwanich ${ }^{30}$, Z.Y. Pei ${ }^{11}$, M.Y. Qi ${ }^{1,3}$, Y.Q. Qi ${ }^{14}$, B.Q. Qiao ${ }^{1,3}$, J.J. Qin ${ }^{7}$, D. Ruffolo ${ }^{30}$, V. Rulev ${ }^{25}$, A. Sáiz ${ }^{30}$, L. Shao ${ }^{14}$, O. Shchegolev $^{25,31}$, X.D. Sheng ${ }^{1,3}$, J.Y. Shi ${ }^{1,3}$, H.C. Song ${ }^{26}$, Yu.V. Stenkin ${ }^{25,31}$, V. Stepanov ${ }^{25}$, Y. Su ${ }^{32}$, Q.N. Sun ${ }^{8}$, X.N. Sun ${ }^{27}$, Z.B.

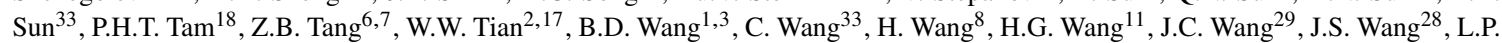
Wang $^{22}$, L.Y. Wang ${ }^{1,3}$, R.N. Wang ${ }^{8}$, W. Wang ${ }^{18}$, W. Wang ${ }^{12}$, X.G. Wang ${ }^{27}$, X.J. Wang ${ }^{1,3}$, X.Y. Wang ${ }^{10}$, Y. Wang ${ }^{8}$, Y.D. Wang ${ }^{1,3}$, Y.J. Wang $^{1,3}$, Y.P. Wang ${ }^{1,2,3}$, Z.H. Wang ${ }^{9}$, Z.X. Wang ${ }^{20}$, Zhen Wang ${ }^{28}$, Zheng Wang ${ }^{1,3,6}{ }^{1, \text { D.M. Wei }}{ }^{13}$, J.J. Wei ${ }^{13}$, Y.J. Wei ${ }^{1,2,3}$, T. Wen $^{20}$, C.Y. $\mathrm{Wu}^{1,3}$, H.R. $\mathrm{Wu}^{1,3}$, S. Wu ${ }^{1,3}$, W.X. Wu ${ }^{8}$, X.F. Wu ${ }^{13}$, S.Q. Xi ${ }^{1,3}$, J. Xia ${ }^{7}, 13$, J.J. Xia ${ }^{8}$, G.M. Xiang ${ }^{2,15}$, D.X. Xiao ${ }^{16}$, G. Xiao ${ }^{1,3}$, H.B. Xiao ${ }^{11}$, G.G. Xin ${ }^{12}$, Y.L. Xin ${ }^{8}$, Y. Xing ${ }^{15}$, D.L. Xu ${ }^{28}$, R.X. Xu ${ }^{26}$, L. Xue ${ }^{22}$, D.H. Yan ${ }^{29}$, J.Z. Yan ${ }^{13}$, C.W. Yang 9 , F.F. Yang ${ }^{1,3,6}$, J.Y. Yang ${ }^{18}$, L.L. Yang ${ }^{18}$, M.J. Yang ${ }^{1,3}$, R.Z. Yang ${ }^{7}$, S.B. Yang ${ }^{20}$, Y.H. Yao ${ }^{9}$, Z.G. Yao ${ }^{1,3}$, Y.M. Ye ${ }^{23}$, L.Q. Yin ${ }^{1,3}$, N. Yin ${ }^{22}$, X.H. You $^{1,3}$, Z.Y. You ${ }^{1,2,3}$, Y.H. Yu ${ }^{22}$, Q. Yuan ${ }^{13}$, H.D. Zeng ${ }^{13}$, T.X. Zeng ${ }^{1,3,6}$, W. Zeng ${ }^{20}$, Z.K. Zeng ${ }^{1,2,3}$, M. Zha ${ }^{1,3}$, X.X. Zhai ${ }^{1,3}$, B.B. Zhang $^{10}$, H.M. Zhang ${ }^{10}$, H.Y. Zhang ${ }^{22}$, J.L. Zhang ${ }^{17}$, J.W. Zhang ${ }^{9}$, L.X. Zhang ${ }^{11}$, Li Zhang ${ }^{20}$, Lu Zhang ${ }^{14}$, P.F. Zhang ${ }^{20}$, P.P. Zhang ${ }^{14}$, R. Zhang ${ }^{7,13}$, S.R. Zhang ${ }^{14}$, S.S. Zhang ${ }^{1,3}$, X. Zhang ${ }^{10}$, X.P. Zhang ${ }^{1,3}$, Y.F. Zhang ${ }^{8}$, Y.L. Zhang ${ }^{1,3}$, Yi Zhang ${ }^{1,13}$, Yong Zhang ${ }^{1,3}$, B. $Z_{\text {Zhao }}^{8}$, J. Zhao ${ }^{1,3}$, L. Zhao ${ }^{6,7}$, L.Z. Zhao ${ }^{14}$, S.P. Zhao ${ }^{13,22}$, F. Zheng ${ }^{33}$, Y. Zheng ${ }^{8}$, B. Zhou ${ }^{1,3}$, H. Zhou ${ }^{28}$, J.N. Zhou ${ }^{15}$, P. Zhou ${ }^{10}$, R. Zhou $^{9}$, X.X. Zhou ${ }^{8}$, C.G. Zhu ${ }^{22}$, F.R. Zhu ${ }^{8}$, H. Zhu ${ }^{17}$, K.J. Zhu ${ }^{1,2,3,6}$ and X. Zuo ${ }^{1,3}$

${ }^{1}$ Key Laboratory of Particle Astrophyics \& Experimental Physics Division \& Computing Center, Institute of High Energy Physics, Chinese Academy of Sciences, 100049 Beijing, China.

${ }^{2}$ University of Chinese Academy of Sciences, 100049 Beijing, China.

${ }^{3}$ TIANFU Cosmic Ray Research Center, Chengdu, Sichuan, China.

${ }^{4}$ Dublin Institute for Advanced Studies, 31 Fitzwilliam Place, 2 Dublin, Ireland.

${ }^{5}$ Max-Planck-Institut for Nuclear Physics, P.O. Box 103980, 69029 Heidelberg, Germany.

${ }^{6}$ State Key Laboratory of Particle Detection and Electronics, China.

${ }^{7}$ University of Science and Technology of China, 230026 Hefei, Anhui, China.

${ }^{8}$ School of Physical Science and Technology \& School of Information Science and Technology, Southwest Jiaotong University, 610031 Chengdu, Sichuan, China.

${ }^{9}$ College of Physics, Sichuan University, 610065 Chengdu, Sichuan, China.

${ }^{10}$ School of Astronomy and Space Science, Nanjing University, 210023 Nanjing, Jiangsu, China.

${ }^{11}$ Center for Astrophysics, Guangzhou University, 510006 Guangzhou, Guangdong, China.

${ }^{12}$ School of Physics and Technology, Wuhan University, 430072 Wuhan, Hubei, China.

${ }^{13}$ Key Laboratory of Dark Matter and Space Astronomy, Purple Mountain Observatory, Chinese Academy of Sciences, 210023 Nanjing, Jiangsu, China.

${ }^{14}$ Hebei Normal University, 050024 Shijiazhuang, Hebei, China.

${ }^{15}$ Key Laboratory for Research in Galaxies and Cosmology, Shanghai Astronomical Observatory, Chinese Academy of Sciences, 200030 Shanghai, China.

${ }^{16}$ Key Laboratory of Cosmic Rays (Tibet University), Ministry of Education, 850000 Lhasa, Tibet, China.

${ }^{17}$ National Astronomical Observatories, Chinese Academy of Sciences, 100101 Beijing, China.

${ }^{18}$ School of Physics and Astronomy \& School of Physics (Guangzhou), Sun Yat-sen University, 519000 Zhuhai, Guangdong, China.

${ }^{19}$ Dipartimento di Fisica dell'Università di Napoli 'Federico II', Complesso Universitario di Monte Sant’Angelo, via Cinthia, 80126 Napoli, Italy.

${ }^{20}$ School of Physics and Astronomy, Yunnan University, 650091 Kunming, Yunnan, China.

${ }^{21}$ D'epartement de Physique Nucl'eaire et Corpusculaire, Facult'e de Sciences, Universit'e de Gen 'eve, 24 Quai Ernest Ansermet, 1211 Geneva, Switzerland.

${ }^{22}$ Institute of Frontier and Interdisciplinary Science, Shandong University, 266237 Qingdao, Shandong, China.

${ }^{23}$ Department of Engineering Physics, Tsinghua University, 100084 Beijing, China.

${ }^{24}$ School of Physics and Microelectronics, Zhengzhou University, 450001 Zhengzhou, Henan, China.

${ }^{25}$ Institute for Nuclear Research of Russian Academy of Sciences, 117312 Moscow, Russia. 
${ }^{26}$ School of Physics, Peking University, 100871 Beijing, China.

${ }^{27}$ School of Physical Science and Technology, Guangxi University, 530004 Nanning, Guangxi, China.

${ }^{28}$ Tsung-Dao Lee Institute \& School of Physics and Astronomy, Shanghai Jiao Tong University, 200240 Shanghai, China.

${ }^{29}$ Yunnan Observatories, Chinese Academy of Sciences, 650216 Kunming, Yunnan, China.

${ }^{30}$ Department of Physics, Faculty of Science, Mahidol University, 10400 Bangkok, Thailand.

${ }^{31}$ Moscow Institute of Physics and Technology, 141700 Moscow, Russia.

${ }^{32}$ Key Laboratory of Radio Astronomy, Purple Mountain Observatory, Chinese Academy of Sciences, 210023 Nanjing, Jiangsu, China.

${ }^{33}$ National Space Science Center, Chinese Academy of Sciences, 100190 Beijing, China. 